AperTO - Archivio Istituzionale Open Access dell'Università di Torino

\title{
A right ventricular outflow tract focus triggering tachycardiomyopathy in a peripartum patient exposed to radiation and chemotherapy
}

This is a pre print version of the following article:

Original Citation:

Availability:

This version is available http://hdl.handle.net/2318/1624231

since 2019-06-01T16:48:28Z

Published version:

DOI:10.2459/JCM.0000000000000481

Terms of use:

Open Access

Anyone can freely access the full text of works made available as "Open Access". Works made available under a Creative Commons license can be used according to the terms and conditions of said license. Use of all other works requires consent of the right holder (author or publisher) if not exempted from copyright protection by the applicable law. 
This is the author's final version of the contribution published as:

Anselmino, Matteo; Montali, Nicolò; Gaita, Fiorenzo. A right ventricular outflow tract focus triggering tachycardiomyopathy in a peripartum patient exposed to radiation and chemotherapy. JOURNAL OF

CARDIOVASCULAR MEDICINE. 18 (Suppl 1) pp: 187-189.

DOI: 10.2459/JCM.0000000000000481

The publisher's version is available at:

http://Insights.ovid.com/crossref?an=01244665-201701001-00040

When citing, please refer to the published version.

Link to this full text:

http://hdl.handle.net/2318/1624231 


\section{A right ventricular outflow tract focus triggering}

\section{tachycardiomyopathy in a peripartum patient exposed}

\section{to radiation and chemotherapy}

Matteo Anselmino, MD PhD, Niccolò Montali, MD, Fiorenzo Gaita, MD Prof

Division of Cardiology, Department of Medical Sciences

ñCittà della Salute e della Scienzaò Hospital,

University of Turin, Italy

Number of words: 855, 1 Figure, 5 references

Corresponding author: Matteo Anselmino

Address: C.so A.M. Dogliotti, 14 10126, Torino, Italy

Telephone number: (39)-0116709598

Fax: (39)-0112369598

Email: matteo.anselmino@unito.it 


\section{Case report}

A 38 years old patient affected by asymptomatic (NYHA I) mild left ventricle systolic dysfunction (ejection fraction, EF 50\%) secondary to previous chest ionizing radiation and chemotherapy exposure was referred to our Institution due to functional class (NYHA IV) and left EF worsening (30\%) two months after her second pregnancy and concomitant to numerous (>35000/24 h) monomorphic ventricular ectopic beats (VEBs) from the right ventricle outflow tract (RVOT).

The patient had a ganglioneuroblastoma treated by high dose radiotherapy and chemotherapy (doxorubicin, vincristine, cyclophosphamide) when she was two years old. Since then a stable mild left ventricle systolic dysfunction (EF 50\%) persisted, not requiring cardiac therapy as she was completely asymptomatic. She was a former smoker until the age of 36 when she stopped as was giving birth to his first child. During the first pregnancy cardiac function remained stable.

A year later she remained pregnant for a second time. During the second trimester she complained dyspnea (NYHA III) and systolic cardiac function deteriorated to EF 40\%, also documented by Brain Natriuretic Peptide (BNP) increase beyond reference limit. She was therefore diagnosed acute decompensated chronic heart failure and was given furosemide 25 mg bid, bisoprolol $1.25 \mathrm{mg}$ bid and nocturnal transdermal nitrates. Cardiac conditions improved and caesarean section was performed at 34 weeks due to obstetric indication. Following delivery the patient was given enalapril $5 \mathrm{mg}$ bid, bisoprolol was up titrated to $5 \mathrm{mg}$ q.d. and furosemide was stopped due to complete symptoms relief and documentation of EF restoration (to basal EF 50\%) and. Two months later, however, she presented with palpitations due to numerous monomorphic VEBs originating from the RVOT (Figure 1a), 
unresponsive to high dose beta-blocker treatment (first bisoprolol $5 \mathrm{mg}$ q.d, then metoprolol $100 \mathrm{mg}$ bid). In fact, in the following month her clinical conditions rapidly deteriorated (NYHA IV, EF 30\%) despite maximal CHF therapy.

At admission, accordingly to history of chest radiation exposure, even though the patient had never complained angina, a coronary angiography was performed and revealed severe calcific proximal right coronary artery stenosis that was treated by percutaneous drug eluted stenting. As VEBs did not decrease even after revascularization she underwent catheter ablation of the RVOT focus (Figure 1b: right and left lateral Carto, Biosense Webster, mapping system views localizing the ablated spot, red dots, at the septal portion of the RVOT; colours relate to activation during VEBs with red being early and blue-violet late activation). At 3month follow-up ECG (please note incomplete left bundle branch block, Figure 1c) and 24Holter recording (288 VEBs) documented complete resolution of the arrhythmia; at the same time symptoms and EF were restored to basal (EF 50\%, NYHA I).

\section{Discussion}

Cardiomyopathy during pregnancy is uncommon but potentially catastrophic to maternal health, accounting for up to $11 \%$ of maternal deaths ${ }^{1}$. Peripartum cardiomyopathy (PPCM) is currently defined based on the presence of three criteria: I - development of symptomatic heart failure toward the end of pregnancy or in the months following delivery (usually 3-4 months); II - the absence of an identifiable cause of heart failure; and III - left ventricular systolic dysfunction ( $\mathrm{LV}$ ejection fraction $<45 \%$ on echocardiogram $)^{2}$. The cause of PPCM has remained unclear. Recent research, however, suggests that PPCM could be a vascular disease (triggered by hormonal changes in late pregnancy) in which apoptosis in endothelial cells plays a central role 3 . On the other side arrhythmias are the most common cardiac complication encountered during pregnancy in women with and without structural heart 
disease $^{4}$. The haemodynamic changes of pregnancy and postpartum have been well studied 5 and volume overload is the major contributor to arrhythmias development.

In the present patient we observed two distinct haemodynamic deteriorations: whether the first (II trimester of the last pregnancy) seems due to worsening of a pre-existent left ventricle systolic dysfunction in a radiated heart or to PPCM, the second (two months after delivery) appears , instead, to be a tachycardiomyopathy due to numerous VEBs. If VEBs just happened to occur during postpartum or if the later functioned as trigger remains unanswered; in any case the clinical situation was worsened by the underlying radiation and chemotherapy exposed heart. In fact, we believe the calcific proximal coronary stenosis a "silent" bystander contributing to worsening of symptoms during both haemodynamic deteriorations but probably not the cause of either of the two. 
Figure 1.
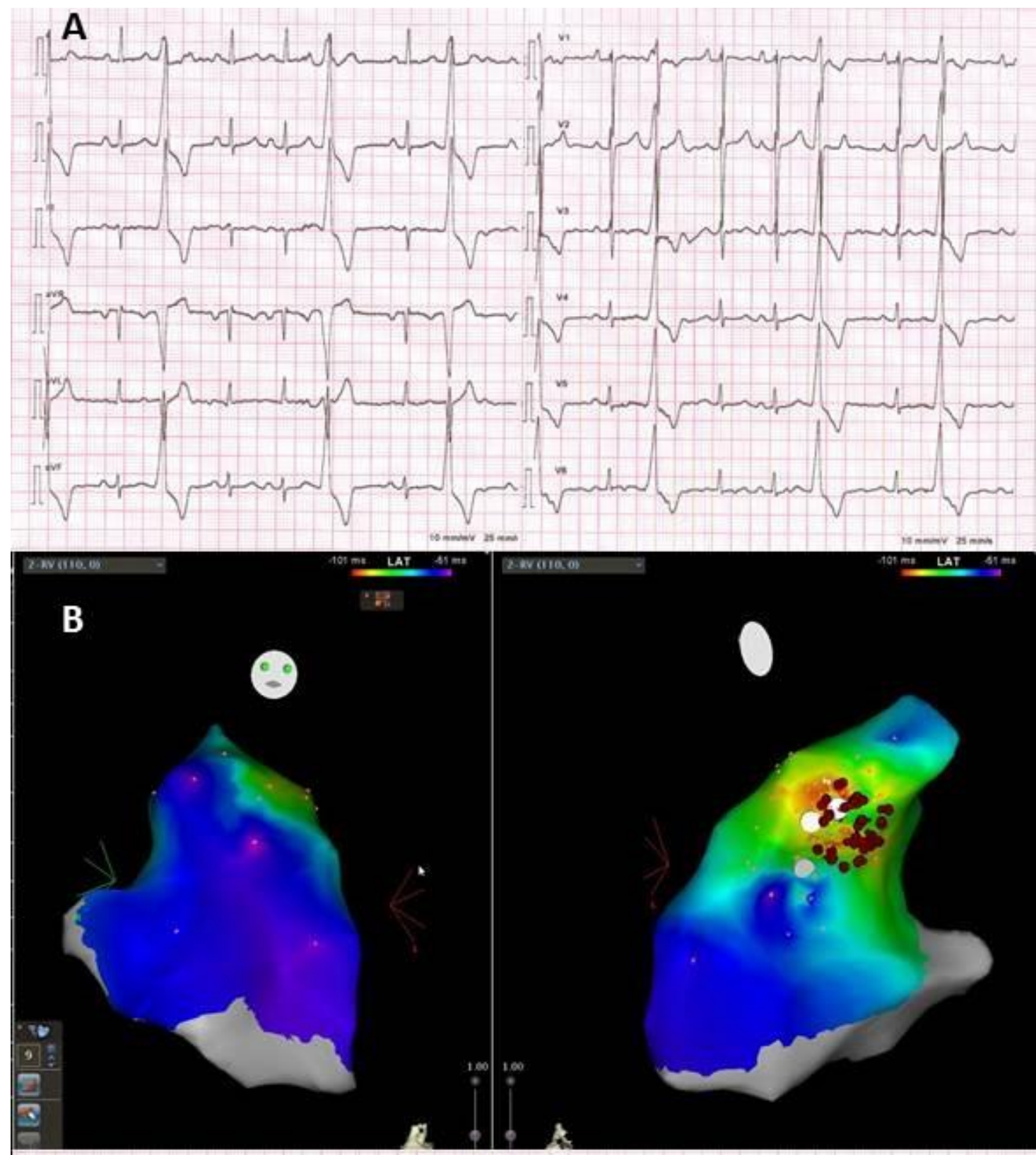

C

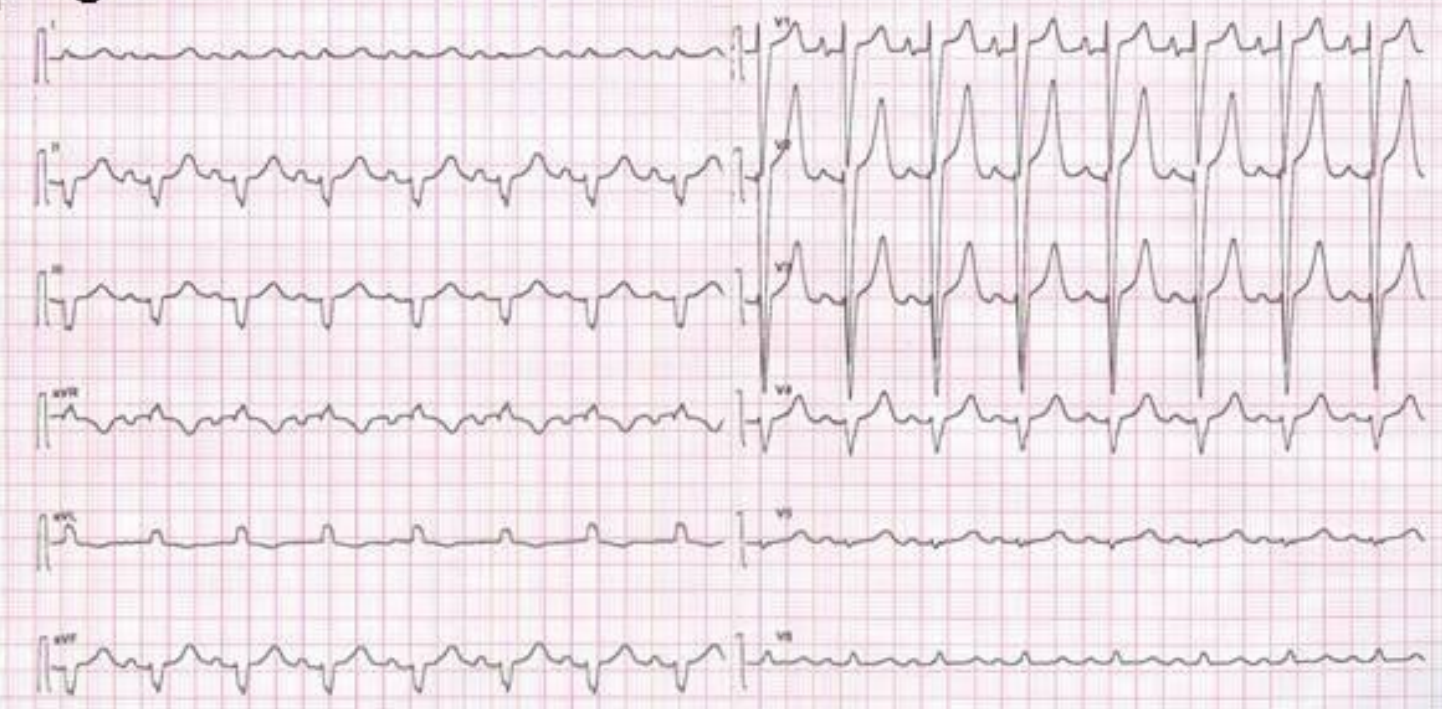




\section{References}

1 C.J. Berg, W.M. Callaghan, C. Syverson, Z. Henderson. Pregnancy-related mortality in the United States, 1998 to 2005. Obstet Gynecol 2010; 116(6):1302-1309

2 K. Sliwa, D. Hilfiker-Kleiner, M.C. Petrie, et al. Current state of knowledge on aetiology, diagnosis, management, and therapy of peripartum cardiomyopathy: a position statement from the Heart Failure Association of the European Society of Cardiology Working Group on peripartum cardiomyopathy. Eur J Heart Fail 2010; 12(8):767-778

3 D. Hilfiker-Kleiner, K. Sliwa, H. Drexler. Peripartum cardiomyopathy: recent insights in its pathophysiology. Trends Cardiovasc Med 2008; 18(5):173-9

4 Siu SC, Sermer M, Colman JM, et al. Prospective multicenter study of pregnancy outcomes in woman with heart disease. Circulation 2001; 104(5):515-21

${ }^{5}$ Robson SC, Dunlop W, Moore M, Hunter S. Combined Doppler and echocardiographic mesurement of cardiac output: theory and application in pregnancy. Br J Obstet Gynaecol 1987; 94(11):1014-27 